\title{
Uso de implantes zigomáticos na fixação de próteses bucomaxilofaciais
}

\author{
Use of zygomatic implants in the fixacion of maxillofacial prothesis
}

\section{RESUMO}

A prótese bucomaxilofacial tem como objetivo a reabilitação de indivíduos com deformidades craniofaciais, decorrentes de traumatismos, más formações congênitas ou tumores. Muitas pesquisas foram realizadas para melhorar a estabilidade e retenção das próteses em pacientes mutilados. Com o advento da osseointegração, a prótese bucomaxilofacial sofreu um grande avanço tornando possível a realização de trabalhos protéticos com estabilidade, qualidade estética e resultados previsíveis. Esse trabalho faz uma revisão de literatura a respeito do uso de implantes zigomáticos na fixação de próteses bucomaxilofaciais.

Palavras-chave: prótese maxilofacial, Implante, qualidade de vida.

\section{ABSTRACT}

The maxillofacial prosthetic rehabilitation aims of individuals with craniofacial deformities resulting from trauma, congenital malformations or tumors. Many researches have been done to improve the stability and retention of dentures in patients. With the advent of osseointegration, the maxillofacial prosthesis suffered a major advance making possible the realization of prosthetic works with stability, aesthetic quality and predictable results. This paper reviews the literature regarding the use of zygomatic implants in the fixation of maxillofacial prostheses.

Keywords: maxillofacial prosthesis, Maxillofacial Prosthesis Implantation, quality of life.

\section{Marcelo Coelho Goiato* \\ Stefan Fiuza De Carvalho Dekon** \\ Leonardo Viana Pereira*** \\ Lisiane Cristina Bannwart ${ }^{* * * *}$ \\ Rodrigo Antonio De Medeiros***** \\ Glauco Issamu Miyahara****** \\ Daniela Micheline Dos Santos**}

* Professor. Adjunto da Disciplina de Prótese Total - Faculdade de Odontologia de Araçatuba - UNESP.

** Professor Assistente Doutor da Disciplina de Prótese Parcial Fixa - Faculdade de Odontologia de Araçatuba - UNESP

*** Mestre em Odontologia, Área de Prótese - Faculdade de Odontologia de Araçatuba UNESP.

**** Mestre em Odontologia, Área de Prótese Faculdade de Odontologia de Araçatuba

***** Mestre em Odontologia, Área de Prótese

- Faculdade de Odontologia de Araçatuba UNESP

****** Professor. Adjunto da Disciplina de Estomatologia - Faculdade de Odontologia de Araçatuba - UNESP.

** Professora Assistente Doutora da Disciplina de Prótese Parcial Fixa - Faculdade de Odontologia de Araçatuba - UNESP

\section{Endereço para correspondência:}

Marcelo Coelho Goiato

UNESP - Faculdade de Odontologia de Araçatuba - Departamento de Materiais Odontológicos e Prótese José Bonifácio, 1193, Araçatuba - SP Brazil, 16015-050

Email: goiato@foa.unesp.br

Enviado em: 3-9-2012

Aceito em: 24-4-2014 


\section{INTRODUÇÃO}

Apesar dos avanços na cirurgia plástica, sempre haverá necessidade de se reabilitar, por meio de próteses bucomaxilofaciais, pacientes mutilados acometidos por patologias ou traumas. A reabilitação bucomaxilofacial permite o retorno de muitos pacientes com defeitos orofaciais ao convívio social. ${ }^{15} \mathrm{~A}$ reabilitação protética de defeitos faciais tem como objetivo, a restauração estética e restabelecimento da auto estima de pacientes mutilados. ${ }^{13}$

Diversas técnicas têm sido descritas para tratar a atrofia maxilar, incluindo o uso de implantes em ângulo na região paranasal, implantes em apófise pterigoide, enxertia do assoalho do seio maxilar, implantes curtos, largos e implantes zigomáticos (IZ). A utilização do IZ após a cirurgia de tumor com ressecção do maxilar óssea, trauma, defeitos congênitos, insucesso com enxerto ósseo autógeno, ferimentos causados por armas de fogo, e em pacientes que se recusam ósseo autógeno. O enxerto é valioso no fornecimento da área zigomática e desempenham um papel fundamental na forma do terço médio da face e em reabilitações orais ${ }^{10}$. Implantes zigomáticos ainda podem ser considerados uma alternativa relevante.

Reabilitar pacientes portadores de deformidades craniofaciais, de origem congênita ou adquirida, tem sido um desafio para as equipes multidisciplinares. Visto a dificuldade de fixação de tais próteses uma solução é a utilização de adesivos, porém o uso destes está associado à dermatite, reações alérgicas e perda de aderência e deslocamento da mesma. ${ }^{12,26,40}$

A utilização de implantes osseointegrados como componente de sustentação de próteses orais e maxilofaciais proporcionou uma nova alternativa de reabilitação para tais pacientes ${ }^{4}$. A retenção por implantes permitem maior estabilidade protética, fornecendo ao paciente maior conforto. Próteses bucomaxilofaciais implanto suportadas têm sido utilizadas satisfatoriamente na reconstrução de orelhas, defeitos nasais e oculares, e defeitos complexos envolvendo várias estruturas craniofaciais. ${ }^{1,2,3,12,14}$

A localização e a orientação dos implantes craniofaciais é fundamental para alcançar o sucesso do resultado. O desenvolvimento da fixação zigomática veio com o objetivo de solucionar os casos de pacientes vítimas de traumas ou cirurgias de remoção de tumores, onde existe grande perda das estruturas maxilares. ${ }^{19,39}$

Estudos indicam a utilização de fixações zigomáticas para a reabilitação de pacientes em várias situações, como atrofia severa de maxila, grandes defeitos maxilares e para casos de ressecção maxilar devido a tumores. ${ }^{9}, 11,17,18,27,28,32$

Sartori et al. ${ }^{30}$ realizaram um estudo de evolução da satisfação dos pacientes reabilitados com implantes zigomáticos. Metade dos pacientes respondeu que estão completamente satisfeitos, enquanto a outra metade está satisfeita, porém com algumas reclamações. As reclamações estão relacionadas com estética, fonética e desconforto durante a mastigação. ${ }^{30}$

O objetivo do trabalho é realizar uma revisão do uso de implantes zigomáticos para pacientes com deformidades craniofaciais.

\section{REVISÃO DE LITERATURA}

A tentativa do ser humano de restaurar artificialmente a face surgiu na época das antigas civilizações. Escavações arqueológicas têm revelado múmias egípcias com olhos, 
nariz e orelhas artificiais. Os povos antigos restauravam os olhos das múmias com pedras preciosas como símbolo de devoção aos deuses. ${ }^{14,29,36}$

Desde a Revolução Industrial os avanços científicos e tecnológicos vêm aumentando em progressão geométrica, de forma a possibilitar novas descobertas e aprimoramento dos materiais e das técnicas existentes. Utilizando-se de novos materiais (resinas, silicones, biomateriais e implantes osseointegrados), associados às técnicas cirúrgicas plásticas e/ ou reparadoras, a especialidade tem aperfeiçoado a confecção de próteses cada vez mais estéticas e biocompatíveis, facilitando o uso pelo paciente e a sua reintegração social, fazendo com que em alguns casos até mesmo retorne as suas atividades normais. ${ }^{33}$

A implantodontia surgiu da necessidade de substituir elementos dentários perdidos, em uma tentativa de recuperar estética e função mastigatória. Dessa forma, devido a sua alta taxa de sucesso, esse conceito foi transferido para reabilitações de deformidades faciais. O primeiro uso de implantes extraorais para reconstrução maxilofacial foi realizado por Branemark em 1979 para retenção de uma prótese auricular. Desde então, os implantes extraorais tornaram-se uma alternativa para reconstrução protética de defeitos maxilofaciais, sendo bem documentada. ${ }^{33}$

Os implantes extra-orais são muito importantes para pacientes com malformações, traumas ou acometidos por patologias como o câncer, e precisam repor partes anatômicas, total ou parcialmente atingidas. Com diferentes tamanhospodendo serinserido na maxila, osso temporal, osso frontal e osso zigomático. Estruturas metálicas para retenção da prótese de silicone, representa uma alternativa commaior sucesso do que as técnicas convencionais. ${ }^{16}$ Melhor retenção e adaptação, faz com que as próteses bucomaxilofaciais adquiram novas perspectivas, eliminando ou minimizando a necessidade de adesivos quando utiliza-se retentores mecânicos tipo clipes ou magnetos, evitando transtorno com o uso de adesivos e os constrangimentos decorrentes do desprendimento das próteses em público. Os implantes zigomáticos servem como posicionadores e permitem um melhor assentamento para o aparelho protético. ${ }^{16}$

A utilização de implantes devidamente confeccionados e instalados com técnicas cirúrgicas corretas é uma boa alternativa para a confecção de pilares de sustentação das próteses bucomaxilofaciais, conferindo ao paciente a possibilidade de colocação e remoção destas de maneira rápida e simples, uma melhor retenção, estabilidade e estética. ${ }^{4,24}$

De sua própria experiência com base em pesquisas com animais e experiências humanas, PI Branemark, sabendo que a introdução de um implante no seio maxilar não compromete necessariamente a saúde,considerou o uso do osso zigomático como um ponto de ancoragem para reabilitação protética em pacientes com hemimaxilectomia, bem como para outros defeitos. Como estas reconstruções foram bem sucedidos e com boa estabilidade a longo prazo destes implantes, em 1997, Branemarkdesenvolveu um específico implante chamado o 'ImplantesioZigomáticos' para fornecer soluções fixas, mesmo quando as condições para a inserção do implante eram pobres na maxila posterior. Este desenvolvimento tecnológico ofereceu novas alternativas aos procedimentos de enxerto ósseo ou de levantamento de seio, que envolvem uma cirurgia pouco invasiva. ${ }^{34}$

Um relatório sobre a utilização de implantes zigomáticos instaladosna cavidade nasal e seio maxilar foi publicado por Branemark em 1984. ${ }^{20}$ Desde então, vários trabalhos têm sido publicados a respeito do desempenho clínico dos implantes zigomáticos $5,20,21,25,31,37$.Foi 
projetado para oferecer ancoragem óssea máxima. ${ }^{8,20}$ Está disponível em oito comprimentos que variam de 30 a 52,5mm. As indicações primárias para estes são maxilas atróficas e pacientes submetidos à maxilectomia ${ }^{20,21,23,25}$ ou que possuem deformidades nasais.

Implantes zigomáticos associados aos convencionais para casos de maxila atrófica são indicados para situações limitadas. É importante considerar que, embora seja ainda um procedimento invasivo porque envolve anestesia geral, este tratamento reduz o número de procedimentos cirúrgicos, o custo, o número de sessões clínicas, e a duração do tratamento. A alta satisfação e taxa de sucesso mostra que é uma alternativa importante para este tratamento. A utilização de implantes zigomáticos em pacientes com atrófica maxila é previsível e confiável. Mas se houver complicações este implantes são facilmente removidos. Além disso, os pacientes demonstram satisfação com seus implantes e próteses. ${ }^{30}$

A utilização de implantes zigomáticos está mais bem descrito na literatura para pacientes com deformidades nasais. ${ }^{6,7}$ Este fator se deve à localização da prótese. Porém, os mesmos podem ser utilizados para retenção de próteses maxilares obturadoras. ${ }^{12,20,22,35}$

A taxa de sucesso de implantes zigomáticos é de $65 \%$ a $75 \%$ após a retirada do tumor e de $71 \%$ com acompanhamento de 4 anos. ${ }^{21,38}$ As causas de falhas dos implantes zigomáticos incluem uma excessiva força de alavanca, infecção e recidiva do tumor. ${ }^{21}$

\section{CONCLUSÃO}

Os implantes zigomáticos proporcionam melhor retenção e adaptação, fazendo com que as próteses bucomaxilofaciais adquiram novas perspectivas. Eles permitem orientação e suporte para o aparelho protético. Está bem relatado na literatura o sucesso do tratamento, surgindo como possibilidade para pacientes que possuem deformidades craniofaciais.

\section{REFERÊNCIAS}

1. Abu-Serriah MM, McGowan DA, Moos KF, Bagg J. Extra-oral endosseous craniofacial implants: current status and future developments. Int J Oral MaxillofacSurg 2003; 32:452-458.

2. Abu-Serriah MM, McGowan DA, Moos KF, Bagg J. Extra-oral endosseous craniofacial implants and radiotherapy. Int J Oral MaxillofacSurg 2003; 32:585-592.

3. Abu-Serriah MM, McGowan DA, Moos KF, Bagg J. Outcome of extra-oral craniofacial endosseous implants. Br J Oral MaxillofacSurg 2001; 39:269-275.

4. Antunes AA. Carvalho RWF, Neto AL, Loretto NRM, Silva EDO. Utilização de implantes ósseointegrados para retenção de próteses buco-maxilo-faciais: revisão da literatura. RevCirTraumatol Buco-Maxilo-fac 2008; 8:09-14.

5. Bedrossian E, Stumpel LJ 3rd. Immediate stabilization at stage II of zygomatic implants: rationale and technique. J Prosthet Dent 2001; 86:10-14.

6. Block MS, Salinas T. Reconstruction of a nasomaxillary defect with traditional and infraorbitalzygomaticus implants: report of a case. J Oral MaxillofacSurg 2002; 60:1362-1366.

7. Bowden JR, Flood TR, Downie IP. Zygomaticus implants for retention of nasal prostheses after rhinectomy. Br J Oral MaxillofacSurg 2006; 44:54-56.

8. Branemark PI, Adell R, Albrektsson T, Lekholm U, Lindstrom J, Rockler B. An experimental and clinical study of osseointegrated implants penetrating the nasal cavity and maxillary sinus. J Oral MaxillofacSurg 1984;42:497-505.

9. Brånemark PI, Gröndahl K, Ohrnell LO, Nilsson P, Petruson B, SvenssonB, et al. Zygoma fixture in the management of advanced atrophy of the maxilla: technique and long-term results. Scand J PlastReconstrSurg Hand Surg 2004;38:70-85.

10. Chrcanovic BR, Pedrosa AR, Custódio AL. Zygomatic implants: a critical review of the surgical techniques. Oral MaxillofacSurg 2012; 25: [Epub ahead of print].

11. Duarte LR, Nary Filho H, Francischone CE, Francischone Jr. CE, Viana A. Fixações zigomáticas: uma excelente alter- 
nativa cirúrgica para a maxila severamente reabsorvida. Revisão de literatura e estágio científico atual. Implant News 2004;1:477-486.

12. Ethunandan M, Downie I, Flood T. Implant-retained nasal prosthesis for reconstruction of large rhinectomy defects: the Salisbury experience. Int J Oral MaxillofacSurg 2010; 39:343-349.

13. Filié Haddad M, Coelho Goiato M, Micheline Dos Santos D, Moreno A, Filipe D’almeida N, Alves Pesqueira A. Color stability of maxillofacial silicone with nanoparticle pigment and opacifier submitted to disinfection and artificial aging. J Biomed Opt 2011;16:095004.

14. Flood TR, Russell K. Reconstruction of nasal defects with implant retained nasal prosthesis. Br J Oral MaxillofacSurg 1998; 36:1-5.

15. Goiato MC, Pesqueira AA, dos Santos DM, Zavanelli AC, Ribeiro Pdo P. Color stability comparison of silicone facial prostheses following disinfection. J Prosthodont2009; 18:242-244.

16. Goiato MC, Tabata LF, Archangelo CM. Uso de implantes osseointegrados associados a sistemas de renteção nas reabilitações com prótese bucomaxilofacial: revisão de literatura. Pesq BrasOdontopedClinIntegr 2007; 7:331-336.

17. Hirsch JM, Ohrnell LO, Henry PJ, Andreasson L, Brånemark PI, Chiapasco M, et al. A clinical evaluation of the Zygoma fixture: one year of follow-up at 16 clinics.J Oral Maxillofac Surg. 2004;62:22-29.

18. Hu YJ, Hardianto A, Li SY, Zhang ZY, Zhang CP. Reconstruction of a palatomaxillary defect with vascularized iliac bone combined with a superficial inferior epigastric artery flap and zygomatic implants as anchorage. Int J Oral MaxillofacSurg 2007;36:854-857.

19. Jensen OT, Brownd C, Blacker J. Nasofacialorostheses supported by osseointegrated implants. Int J Oral Maxilofac Implants 1992; 7: 203-211.

20. Kreissl ME, Heydecke G, Metzger MC, Schoen R.Zygoma implant-supported prosthetic rehabilitation after partial maxillectomy using surgical navigation: a clinical report. J Prosthet Dent 2007;97:121-128.

21. Landes CA. Zygoma implant-supported midfacial prosthetic rehabilitation: a 4-year follow-up study including assessment of quality of life. Clin Oral Implants Res 2005;16:313-325.

22. Leles CR, Leles JL, de Paula Souza C, Martins RR, Mendonça EF.Implant-supported obturatoroverdenture for extensive maxillary resection patient: a clinical report.J Prosthodont 2010; 19:240-244.

23. Malevez C, Daelemans P, Adriaenssens P, Durdu F. Use of zygomatic implants to deal with resorbed posterior maxillae. Periodontol 2000 2003; 33:82-89.

24. Nascimento DS, Oliveira GMF, Wanderley TKC, Loretto NRM, Gerbi MEMM. Contribuição da implantologia para a prótese buco-maxilo-facial. Odontol ClínCient 2011; 10: 417-419.

25. Parel SM, Branemark PI, Ohrnell LO, Svensson B. Remote implant anchorage for the rehabilitation of maxillary defects. J Prosthet Dent 2001;86: 377-381.

26. Parel SM. Diminishing dependence on adhesives for retention of facial prosthesis. J Prosthet Dent 1980; 43:552-560.

27. Peñarrocha-Diago M, Uribe-Origone R, Rambla-Ferrer J, Guarinos-Carbó J. Fixed rehabilitation of a patient with hypohidrotic ectodermal dysplasia using zygomatic implants. Oral Surg Oral Med Oral Pathol Oral RadiolEndod. 2004;98:161-165.

28. Pham AV, Abarca M, De Mey A, Malevez C. Rehabilitation of a patient with cleft lip and palate with an extremely edentulous atrophied posterior maxilla using zygomatic implants: case report. CleftPalateCraniofac J2004;41:571-574.

29. Rezende JRV, Oliveira JAP, Dias RB. Prótese buco maxilo facial: conceitos básicos e práticas de laboratório. São Paulo: Sarvier; 1986.

30. Sartori EM, Padovan LE, de Mattias Sartori IA, Ribeiro PD Jr, Gomes de Souza Carvalho AC, Goiato MC.Evaluation of satisfaction of patients rehabilitated with zygomatic fixtures. J Oral MaxillofacSurg2012; 70:314-319.

31. Schmidt BL, Pogrel MA, Young CW, Sharma A. Reconstruction of extensive maxillary defects using zygomaticus implants. J Oral MaxillofacSurg 2004;62:82-89.

32. Schramm A, Gellrich NC, Schimming R, Schmelzeisen R. Computerassisted insertion of zygomatic implants (Brånemark System) after extensive tumor surgery. Mund Kiefer Gesichtschir. 2000;4:292-295.

33. Scolozzi P, Jaques B. Treatment of midfacial defects using prostheses supported by ITI dental Implants. PlastReconstrSurg 2004;114:1395-1404.

34. Sharma A, Rahul GR. Zygomaticimplants / fixture - a systematic review.J Oral Implantol 2012; 16: [Epub ahead of print]

35. Shirota T, Shimodaira O, Matsui Y, Hatori M, Shintani S.Zygoma implant-supported prosthetic rehabilitation of a patient with a maxillary defect. Int J Oral MaxillofacSurg 2011; 40:113-117.

36. Simões FG, Reis RC, Dias RB. The specialty of bucomaxillofacial prosthesis and its performance in Dentistry. RSBO 2009; 6:327-331.

37. Uchida Y, Goto M, Katsuki T, Akiyoshi T. Measurement of the maxilla and zygoma as an aid in installing zygomatic implants. J Oral MaxillofacSurg 2001;59:1193-1198.

38. Weingart D,Schilli W,Strub JR. Preprosthetic surgery and implantology. The effect of oral implantology on preprosthetic 
surgery in edentulous patients.SchweizMonatsschrZahnmed 1992;102:1074-1085.

39. Weischer T, Schettler D, Mohr C. Titanium Implants in the Zygoma as Retaining Elements after Hemimaxillectomy. Int J Oral Maxillofac Implants 1997;12:211-214.

40. Wolfaardt J, Gehl G, Farmand M, Wilkes G. Indications and methods of care for aspects of extra oral osseointegration. Int J Oral MaxillofacSurg 2003; 32:124-131. 